\title{
The Gamma-Gamma Channel Model - A Survey
}

\author{
Kulvir Kaur ${ }^{1}$, Rajan Miglani1* and Jagjit Singh Malhotra ${ }^{2}$ \\ 'Department of Electronics and Communication systems, Lovely Professional University, Phagwara - 144411 \\ Punjab, India; sidhhukulvir18@gmail.com, rajan.16957@lpu.co.in \\ 2Department of ECE, DAVIET, Jalandhar - 144008, Punjab, India; jmalhotra292@gmail.com
}

\begin{abstract}
Background: In Free space optical communication medium, the received signal envelope is affected by various impairments. The most dominating effect is turbulence induced fading. To model the turbulence there are fading channel models. Findings: In this paper the Gamma-gamma fading model is reviewed which gives impressive results under all types of turbulence conditions. The Gamma-gamma channel model statistics and its performance with spatial diversity techniques under correlation are reviewed. It has been shown that the Gamma-gamma channel model is able to perform better under correlation than other fading channel models. Improvements: The performance analysis of Gamma-gamma channel under pointing errors and its working with recent techniques in which FSO systems works with RF systems (Hybrid RF/FSO system) are also included in this paper.
\end{abstract}

Keywords: Gamma-Gamma Channel Model, FSO (Free Space Optics), RF (Radio Frequency)

\section{Introduction}

Free Space Optical (FSO) communication is a wireless communication technique which uses light as carrier. It has various those attributes over RF (Radio Frequency) wireless communication which makes it more attractive. The data rate provided by FSO is upto $10 \mathrm{Gbps}$ which is very high as compared to $\mathrm{RF}$ technology ${ }^{1}$. It uses IR and visible range of electromagnetic spectrum which is license free ${ }^{2}$. This technique requires a line of sight path to provide communication between sources to destination and has narrow bandwidth so it provides high security ${ }^{2}$. The FSO has various good properties; on the other side its received signal envelope impaired by some channel circumstances. There are various losses which degrades the channel performance like atmospheric losses (rain, fog, snow), misalignment losses (beam wander and building sway) and turbulence induced fading ${ }^{3,4}$. The turbulence induced fading is the most dominating performance degrade phenomenon in $\mathrm{FSO}^{4}$. To explain the turbulence induced fading Kolomogorov theory has been proposed ${ }^{5}$ To analyze the performance of FSO link channel various models has been proposed which comes under channel modeling. To reduce the effect of channel losses various mitigating techniques are used like modulation, channel coding and diversity techniques. This survey paper has

Source

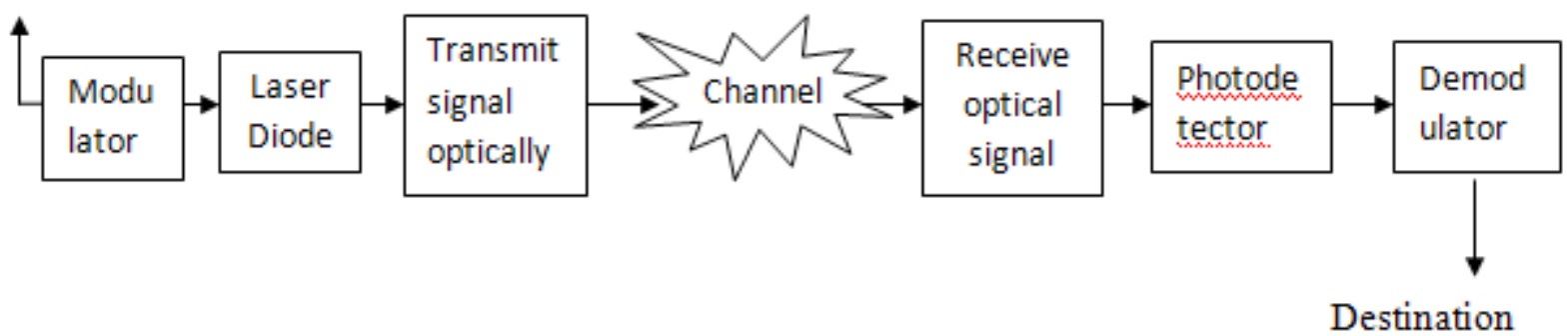

Figure 1. Basic block diagram of Free space optical communication system. 
been divided into six sections. Section 2 focuses on study of channel modeling. The Gamma-gamma channel statistics is considered in section 3. The section 4 describes the performance of Gamma-gamma model under various effects. Section 5 contains current and future trends associated with Gamma-gamma channel model. And this paper is concluded with section 6.

\section{Channel Modeling}

To measure the extent of turbulence losses many models are there like Lognormal, Rayleigh, Rician, Negative exponential distribution, Gamma-gamma and recently proposed Malaga(M) distribution.

The Lognormal model is considered and found that it has weak turbulence condition if long propagation path is considered ${ }^{6}$. There is another model, $\mathrm{K}$-distribution ${ }^{\mathrm{z}}$ which has strong turbulence condition. The Negative exponential has been proposed and shown that it has very strong turbulence condition because its PDF (Probability Density Function) gives appropriate results in negative region ${ }^{-}$. There are some other models based on doubly stochastic theor $y^{8}$ like Gamma-gamma, Malaga distribution which works under weak to strong turbulence conditions.

Table 1. Various Channel models with Turbulence

\begin{tabular}{|l|l|}
\hline Channel Model & Turbulence \\
\hline Lognormal $^{\underline{6}}$ & Weak \\
\hline Negative exponential $^{\underline{8}}$ & Very strong \\
\hline K-distribution $^{\underline{7}}$ & strong \\
\hline Gamma-gamma $^{\underline{8}}$ & Weak to strong \\
\hline
\end{tabular}

\section{Gamma-Gamma Model Statistics}

The Gamma-gamma scintillation model which is based on Doubly Stochastic theory is widely accepted in literature ${ }^{8}$. As we know Gamma-gamma model has weak to strong turbulence condition so the PDF (Probability Density Function) of its intensity I is product of two gamma random variables which represents fluctuations from small and large turbulence. The two random variable are $\mathrm{X}$ and $\mathrm{Y}$ and the received intensity $\mathrm{I}$ is:

$$
I=X Y
$$

The PDF of I is given in equation 2.

$$
\mathrm{p}(I)=\frac{\frac{2(\alpha \beta)((\alpha+\beta))}{2}}{\Gamma^{(\alpha)} \Gamma(\beta)} I^{((\alpha+\beta) / 2)-1} \mathrm{~K}_{\alpha-\beta}(2 \sqrt{\alpha \beta I}), \mathrm{I}>0
$$

I is irradiance

$\Gamma($.$) is gamma function$

$K(\alpha, \beta)$ is Bessel function of second order.

The $\alpha$ and $\beta$ are numbers of small and large turbulence cells and given by equation no. 3 and 4 respectively:

$$
\alpha=\frac{1}{\exp \left[\frac{0.49 \sigma^{2}}{\left.\left(\mathbb{Z}_{1}+1.11 \sigma^{\frac{12}{5}}\right) \frac{7}{1 \frac{7}{6}}\right]-1}\right.}
$$

Where $\sigma^{2}$ represents variance.

The Scintillation Index (SI) which is used to describe the quantity of turbulence for this channel model is given as:

$$
\sigma^{2}=(1 / \alpha)+(1 / \beta)+(1 / \alpha \beta) .
$$

\section{Performance of Gamma- Gamma Model under Various Effects}

The Gamma-gamma distribution shows excellent agreement with all ranges of turbulence if no spatial diversity techniques applied to it. But in order to remove turbulence effect spatial diversity is one of the best mitigating technique. The gamma-gamma distribution shows significant results if spatial diversity techniques have no correlation between sub-channels and it can be easily modified in such scenarios. In the gamma-gamma distribution is used to model the independent gammagamma random variables 9 . The other example of this technique has been demonstrated in where $\alpha-\mu$ distribution (generalized gamma) is used to model independent gamma-gamma variables ${ }^{10}$. Nevertheless, in practical situation the fading correlation between sub-channels is inevitable. In it explained that for large link distance 
the correlation is significant but in some other previous works it degrades the performance of system if simple statistical models are considered $\frac{10,12,13}{}$. However, the closed form is also difficult to achieve in case of Gamma-gamma model with correlation. As in recent the Gamma-gamma channel with correlation under MISO diversity is modeled by Gaussian distribution and it is concluded that the operation requires less power at low BER $\stackrel{14}{ }$. The multivariate Gamma-gamma distribution under correlation of large scale fluctuations and independent small-scale fluctuations is considered and it is concluded that it does not work for most FSO systems $s^{15}$. The $\alpha-\mu$ distribution is then used under the effect of correlation where SIMO diversity is considered and it has been shown that the

Table 2. Performance of Gamma-gamma channel model under various effects

\begin{tabular}{|c|c|c|c|}
\hline $\begin{array}{l}\text { Channel } \\
\text { Model }\end{array}$ & Diversity & Effect & Comments \\
\hline $\begin{array}{l}\text { Gamma- } \\
\text { gamma }^{\underline{14}}\end{array}$ & MISO & $\begin{array}{l}\text { Gaussian } \\
\text { distribution }\end{array}$ & $\begin{array}{l}\text { At low BER, } \\
\text { the system } \\
\text { operation } \\
\text { requires less } \\
\text { power. }\end{array}$ \\
\hline $\begin{array}{l}\text { Gamma- } \\
\operatorname{gamma}^{\underline{16}}\end{array}$ & SIMO & $\begin{array}{l}\alpha-\mu \\
\text { distribution }\end{array}$ & $\begin{array}{l}\text { As diversity } \\
\text { order increases, } \\
\text { the system loses } \\
\text { its accuracy. }\end{array}$ \\
\hline $\begin{array}{l}\text { Gamma- } \\
\operatorname{gamma}^{\underline{17}}\end{array}$ & SIMO & $\begin{array}{l}\text { Padè } \\
\text { approximation }\end{array}$ & $\begin{array}{l}\text { Ineffective } \\
\text { method for low } \\
\text { BER of order } \\
10^{-8} \text {. }\end{array}$ \\
\hline $\begin{array}{l}\text { Gamma- } \\
\text { gamma }^{19}\end{array}$ & MIMO & $\begin{array}{l}\text { Under } \\
\text { pointing } \\
\text { errors }\end{array}$ & $\begin{array}{l}\text { With high } \\
\text { effect of } \\
\text { pointing errors, } \\
\text { FSO system } \\
\text { becomes } \\
\text { unworkable for } \\
\text { BER }>10^{-1} \text {. }\end{array}$ \\
\hline
\end{tabular}

system loses its accuracy as the diversity order increases $\frac{15}{}$. The Pade approximation is used to obtain the PDF from MGF (Moment Generating Function) of Gamma-gamma random variables and it is analyzed that it has poor performance for low BER less than $10^{-8} \frac{16}{16}$. The FSO system performance also affected by pointing errors which comes from misalignment between sender and receivers. The PDF of Gamma-gamma model is derived as Meijer function under effect of pointing errors $s^{18}$. In recent studies the performance of Gamma-Gamma fading channel with MIMO diversity under pointing errors is analyzed and it deduced that as effect of pointing error increases, the FSO system performance becomes speculative for $\operatorname{BER}^{19}\left(\mathbb{\mathbb { C }}>10 \mathbb{\rrbracket}^{\boldsymbol{\top}}(-1)\right)$ in section 4 th.

\section{Current and Future Work with Gamma-Gamma Channel}

The Gamma-gamma channel model is widely accepted model by researchers in present researches and the performance of Gamma-gamma channel model is analyzed under various effects. The gamma-gamma channel is considered with MIMO diversity under Pointing errors and the BER evaluated for both EGC (Equally Gain Combining) and MRC (Maximal Ratio Combining) and it has been concluded that MRC scheme is more invincible than EGC under large effect of pointing errors ${ }^{20}$. The multi-hop property of RF wireless communication in which there are several nodes between sender and receiver is applied in FSO systems also. The multi-hop FSO system to evaluate the outage probability with $\mathrm{K}$ and Gamma- gamma channel was proposed ${ }^{21}$. There is also another technique which known as Hybrid RF/FSO systems in this case the RF system works parallel with FSO system this technique is beneficial because in case of link failure of FSO system the RF provides backup and it is also less affected by turbulence and pointing errors. In recent studies, the Nakagami channel in RF is relayed with Gamma-gamma channel in FSO is proposed where Nakagami channel is established between Source to Relay (S-R) and Gamma-gamma channel is established between Relay to Destination (R-D).

\section{Conclusion}

The FSO communication has some supreme properties which makes it more impressive than RF communication. There are various channel models to demonstrate the working of FSO system in different turbulence scenarios. It is concluded that among all fading models the Gammagamma channel models is more acceptable because it works under all kind of turbulence effects. The other significant property of Gamma-gamma model is that it works under sub-channel correlation effect where other simple channel models are unable to perform better, these attributes of Gamma-gamma channel is reviewed in this paper. 


\section{References}

1. Willebrand HA, Ghuman BS. Fiber optics without fiber. IEEE Spectr. 2001 Aug; 40(8):41-5.

2. Leitgeb E, et al. Current optical technologies for wireless access. Proceeding of International ConTEL; Zagreb, Croatia. 2009 Jun. p. 7-17.

3. Kedar D, Arnon S. Urban optical wireless communication networks: The main challenges and possible solutions. IEEE Commun Mag. 2004 May; 42(5):2-7.

4. Andrews LC, Phillips RL. Laser Beam Propagation through Random Media. 2nd ed. Bellingham, WA, USA: SPIE; 2005.

5. Tatarskii VI, Zavorotnyi VU. Wave propagation in random media with fluctuating turbulent parameters. J Opt Soc Amer A, Opt Image Sci. 1985 Dec; 2(12):2069-76.

6. Andrews LC, Phillips RL, Hopen CY. Laser Beam Scintillation with Applications. Bellingham, WA, USA: SPIE; 2001.

7. Jackman E, Pusey P. Significance of K-distributions in scattering experiments. Phys Rev Lett. 1978 Feb; 40(9):546-50.

8. Al-Habash MA, Andrews LC, Phillips RL. Mathematical model for the irradiance probably density functions of a Laser beam propagating through turbulent media. Opt English. 2001 Aug; 40(8):1554-62.

9. Chatzidiamantis ND, Karagiannidis GK. On the distribution of the sum of Gamma-Gamma variates and applications in RF and optical wireless communications. IEEE Trans Commun. 2011; 59(5):1298-308.

10. Peppas KP. A simple, accurate approximation to the sum of Gamma-Gamma variants and applications in MIMO free-space optical systems. IEEE Phot Technol Lett. 2011; 23(13):839-41.

11. Yang G, Khalighi MA, Bourennane S. Performance of receives diversity FSO systems under realistic beam propagation conditions. IEEE/IET CSNDSP Symposium; 2012.

12. Uysal M, Navidpour SM, Jing L. Error rate performance of coded free-space optical links over strong turbulence channels. IEEE Commun Lett. 2004; 8(10):635-7.
13. Navidpour SM, Uysal M, Kavehrad M. BER performance of free-space optical transmission with spatial diversity. IEEE Trans Wireless Commun. 2007; 6(8):2813-9.

14. Anguita JA, Neifeld MA, Vasic BV. Spatial correlation and irradiance statistics in a multiple-beam terrestrial freespace optical communication link. Appl Opt. 2007 Sep; 46, (26):6561-71.

15. Peppas KP, Alexandropoulos GC, Datsikas CK, Lazarakis FI. Multivariate gamma-gamma distribution with exponential correlation and its applications in radio frequency and optical wireless communications. IET Microwave Ant and Prop. 2011; 5(3):364-71.

16. Yang G, Khalighi MA, Bourennane S, Ghassemlooy Z. Approximation to the sum of two correlated Gammagamma variants and its applications in free-space optical communications. IEEE Wireless Commun Lett. 2012 Dec; 1(6):621-4.

17. Yang G, Khalighi M, Ghassemlooy Z, Bourennane S. Performance analysis $\mathrm{f}$ space-divsity FsO systems over the correlated Gamma-gamma fading channel using Pade approximation method. IET Commun. 2014 Sep; 8(13):2246-55.

18. Gappmair W. Further results on the capacity of free-space optical channels in turbulent atmosphere. IET Commun. 2011; 5(9):1262-7.

19. Bhatnagar MR, Ghassemlooy Z. Performance evaluation of FSO MIMO links in Gamma-gamma fading with pointing error. Proceedings of IEEE International Conference on Communications (ICC); e2015 Jun. London, U. K. p. 1-6.

20. Bhatnagar M, Ghassemlooy Z. Performance analysis of Gamma-gamma Fading FSO MIMO links with pointing errors. J Lightwave Technol. 2016; 34:2158-69.

21. Karagiannidis G, Tsiftsis T, Sandalidis H. Outage probability of relayed free-space optical communication systems. Electron Lett. 2006 Aug; 42(17):994-6. 